\title{
CHANGES IN THE LEFT VENTRICULAR MUSCLE MASS IN ADOLESCENTS
}

\author{
L. Marinov \\ Department of Pediatrics \\ Medical University "Prof. Dr Paraskev Stoyanov"-Varna, Bulgaria
}

\section{SUMMARY}

In the resent short review are presented some of the main reasons for development of left ventricular hypertrophy (LVH). LVH is a multifactor condition caused mainly by arterial hypertension $(\mathrm{AH})$. The changes in the target organs in $\mathrm{AH}$ are a function of the levels of blood pressure (BP) and the duration of the disease. The primary changes because of Arterial Hypertension (AH), independently to its etiology, start in the myocardium of the left ventricle (LV), the arterioles and small arteries of the systemic circulation. The vascular changes in $\mathrm{AH}$ are two types: expression of morphological adaptation to the increased BP, observed in the early stages - most often spasm, causing the subsequent changes in the organs, followed by the complications connected with the increased development of atherosclerosis in different vascular regions leading to ischemic heart disease, brainvessel disease, chronic arterial insufficiency, arrhythmia, sudden death. As a result to the increased requirements to the myocardium in $\mathrm{AH}$, starts adaptive hypertrophy, which at the beginning improves the function of the left ventricle. With the start of the structural changes and remodeling the compensatory abilities of the LV lower and develop heart insufficiency. The increased LV pressure and enlarged muscle mass increase the oxygen needs of the myocardium, which additionally increase the requirements to the coronary blood stream.

Some other additional factors which influence the LVH are discussed like physical development of the children, overweight and obesity. The diagnostic abilities of EKG and EchoCG are discussed for the proving of the LVH. EchoCG and Doppler EchoCG have significantly more abilities, as most informative index for LVH is the index of left ventricular muscle mass.

Key words: left ventricular hypertrophy, index of left ventricular muscle mass, adolescents, EchoCG.

The changes in the target organs in arterial hypertension $(\mathrm{AH})$ are a function of the values of the blood pressure (BP) and the duration of the disease. The primary changes in arterial hypertension (AH), independently to the etiology, start in the myocardium of the left ventricle (LV), arterioles and small arteries in the systemic circulation.

The vascular changes in $\mathrm{AH}$ are two types: expression of morphological adaptation to the increased BP, observed in the early stages - most often spasm, causing the subsequent changes in the organs, followed by the complications connected with the increased development of atherosclerosis in different vascular regions leading to ischemic heart disease, brain-vessel disease, chronic arterial insufficiency, arrhythmia, sudden death. (15). As a result to the increased requirements to the myocardium, starts adaptive hypertrophy, this improves the LV function and is accompanied with structural changes. The increased LV pressure and enlarged muscle mass increases the oxygen needs of the myocardium, which additionally increases the requirements to the coronary blood stream.

The left ventricular hypertrophy ensures the heart to have normal pump function at the presence of constantly increased after - loading from the increased systemic vascular resistance and blood pressure and reduces the left ventricular stress. This is achieved by increase of the size of the myocardial cells, with increased colagenous synthesis and myocardial fibrosis; as a result the heart increases its left ventricular mass and becomes no-effective organ. The hypertrophy leads to reduction of the subendocardial perfusion and increase of the coronary atherosclerosis, which on its turn reduces the coronary blood stream and the oxygen supply.

The changes in the left ventricle pass three stages. First stage - initial answer to the increased myocardial function as a result of increased pressure, which transfers to the second stage - stable adaptive hypertrophy, which improves the LV function and is accompanied with structural changes. The last ones are trying to protect from the development of the third stage - heart insufficiency (4).

In the early stages the ventricular myocit, which is functionally loaded, is stimulated to enlarge its mass by stimulation of the intracellular proto-oncogene mechanism, which provokes DNA- direct protein synthesis. The adaptive hypertrophy of the left ventricle and the connected with it changes, transform some of the 
myocardial needs. The increased left ventricular pressure, the enlarged sizes and diameter of the LV increase the myocardial needs of oxygen, which from its side increases the requirements to the coronary blood stream.

Additional factors which influence LVH are the overweight and obesity associated with $\mathrm{AH}$, as well as the increased viscosity of the blood in hypertension. $(1,3,9)$. In a study from the last months is proved that the highest correlation dependency is demonstrated between overweight and obesity and the geometry and mass of the left ventricle. As higher is the stage of the obesity, the more serious are the following complications (6).

Studies over the LVMM show that it increases in the prehypertensive period of $\mathrm{AH}$, especially in family burdened persons and can be more sensitive factor for subsequent $\mathrm{AH}$ from the levels of BP. (5). There is a correlation between the changes in the carotid arteries and the level of left ventricular hypertrophy in children. The presence of such changes is a serious predictor for already started and developing process of atherosclerotic changes (12).

LVH is accompanied with changes in the left ventricular geometry.

The initial changes are mainly in the septum. When the LVH is mainly because of thickening of the septum, it is asymmetrical. Depending on the ratio thickness/radius of $\mathrm{LV}(\mathrm{h} / \mathrm{r})$, the changes are two types. When this ratio is increased, the left ventricular hypertrophy is described as concentric (thickening of the wall without dilatation of the cavities). When the ration is lowered, the hypertrophy is eccentric.

The LVH is not accompanied with development of new capillaries. The disorders of the coronary microcirculation lead to changes in the distribution of oxygen to the myocardial calls, which is unequal and inadequate and develop degenerative lesions and evolution of the heart insufficiency.

Patients with concentric hypertrophy have stable higher levels of BP in comparison to the patients with normal LV geometry. In patients with $\mathrm{AH}$ and increased levels of aldosteron in plasma, is found significantly higher LVMM and significantly marked concentric hypertrophy, compared to healthy controls.

The classical methods for documentation of the changes in the heart are conventional ECG and EchoCG.

The diagnosis of LVH by electrocardiography is based on voltage criteria or/and presence of repolarization disorders.

The presence of electrical ECG criteria is evidence, in high percentage, for significant increase of LVMM.
ECG is a method with low informative level for the early changes in the heart in adolescents with $\mathrm{AH}(10)$.

The echocardiography (EchoCG) gives the ability, to find the earliest structural and functional changes, as well as their dynamic studying. The most informative for the LV hypertrophy is the index of left ventricular muscle mass (ILVMM).

LVMM is calculated by the corrected of ASE formula of Devereux:

$$
\mathrm{LVM}=0.8\left(1.04\left[(\mathrm{LVDD}+\mathrm{IVS}+\mathrm{PWT})^{3}-\mathrm{LVDD}^{3}\right]\right)+
$$

\section{6}

The index of LMM is calculated LVMM (g)/height $\left(\mathrm{m}^{2.7}\right)$, with which is lowered the influence of age, sex, weight. The left ventricular hypertrophy is defined, for children, when the ILVMM is over $38.6 \mathrm{~g} / \mathrm{m}^{2.7}$. For adults the LVH is defined by ILVMM over $51.0 \mathrm{~g} / \mathrm{m}^{2.7}(11,13)$.

Doppler EchoCG data show that the high SBP correlates with shorter isometric relaxation, higher $\mathrm{V}_{\max }$ and integral of fast LV filling and smaller slope of the increase of the speed during the atria contraction. This probably is a result of minimal structural changes in the myocardium of adolescents and in harder contraction "the phenomenon of ricochet" of the myofibrils is valid, probably because there is still no $\mathrm{Ca}^{++}$overload of the myocite. The higher DBP reliably correlates mainly with the indexes, characterizing the filling of the LV during atria contraction. A higher $\mathrm{V}_{\max }$ is observed and bigger slope, with shorter time for lowering of the speed of the blood stream at the end of diastole. This influences the atria contraction with worsening the profile of filling of the LV in this phase of the diastole, which probably is a result of lowering of the elasticity of myocites, leading to sharp increase of the pressure in LV at the end of diastole and its harder filling. It is considered that these disorders are significant clinical markers for involvement of the left ventricular function. $(8,12)$. In the literature there is data, that even in adolescents with prehypertension, LVH is found in more than $1 / 5$ of the examined $(2,7,14)$.

In conclusion: The changes in the left ventricular geometry and function during childhood are in a process of clarifying. Even in children with prehypertension levels of BP, is found left ventricular hypertrophy, which is surprising. The enlargement of the left ventricular muscle mass, as is seen in the upper exposure, is multifactor and depends on the type of $\mathrm{AH}$, inheritance, physical development of the children. Unfortunately in Bulgaria there are no referent levels for the ILVMM for this age. We consider that it is necessary to develop such indexes for the children's population in our country. 


\section{BIBLIOGRAPHY:}

1. Amin R., Somers V., McConnell K., et al. Activity-adjusted 24-hour ambulatory blood pressure and cardiac remodeling in children with sleep disordered breathing. Hypertension 2008 Jan;51:84-91.

2. Belsha C. W. Left ventricular mass in hypertensive children and adolescents at initial diagnosis. Am.J.Hypert. 2001. Suppl.1: A 240)

3. Border W. L., Kimball T. R., Witt S. A., et al. Diastolic filling abnormalities in children with essential hypertension. Journal of Pediatrics 2007 May; 150(5):503-9)

4. Daniels S. R., Loggie J., Khoury P., et al. Left Ventricular Geometry and Severe Left Ventricular Hypertrophy in Children and Adolescents With Essential Hypertension. Circulation. 1998;97:1907-1911.

5. Juo S. H., Di Tullio M. R., Lin H. F. et al. Heritability of Left Ventricular Mass and Other Morphologic Variables in Caribbean Hispanic Subjects: The Northern Manhattan Family Study. J Am Coll Cardiol, 2005; 46:735-737.
6. Khositseth A., Suthutvoravut U., Chongviriyaphan N. Left Ventricular Mass and Geometry in Obese Children. Asian J. Clin. Nutrition. 2009. 1: 58-64).

7. Kokkinos P., Pittaras A., Narayan P.,et al. Exercise Capacity and Blood Pressure Associations With Left Ventricular Mass in Prehypertensive Individuals. Hypertension. 2007;49:55-61.

8. Luma G., Spiotta R. Hypertension in Children and Adolescents. Am Fam Physician 2006;73:1158-68.

9. Mitsnefes M. M., Kimball T. R., Kartal J., et al. Progression of left ventricular hypertrophy in children with early chronic kidney disease: 2-year follow-up study. Journal of Pediatrics. 2006;149(5):671-675).

10. Rijnbeek P., van Herpen G., Kapusta L., et al. Electrocardiographic Criteria for Left Ventricular Hypertrophy in Children. Pediatric Cardiology. 2008; 29(5): 923-928

11. Seeman T., Gilнk J., Vondrбk K., et al. Regression of Left-Ventricular
Hypertrophy in Children and Adolescents With Hypertension During Ramipril Monotherapy. (Am J Hypertens (2007) 20, 990-996)

12. Sorof J., Alexandrov A., Cardwell G., et al. Carotid Artery Intimal-Medial Thickness and Left Ventricular Hypertrophy in Children With Elevated Blood Pressure. Pediatrics. 2003; 111: 61-66

13. Sorof J., Cardwell G., Franco K., et al. Ambulatory Blood Pressure and Left Ventricular Mass Index in Hypertensive Children. Hypertension. 2002;39:903-908

14. Stabouli S., Kotsis V., Rizos Z., et al. Left ventricular mass in normotensive, prehypertensive and hypertensive children and adolescents. Pediatric Nephrology. Online 15 may 2009.

15. Urbina E. M., Kimball T. R., McCoy C. E., Khoury P. R., Daniels S. R., Dolan L. M. Youth with obesity and obesity-related Type 2 diabetes demonstrate abnormalities in carotid structure and function. Online. To be published in Circulation 2009.

\section{Corresponding Author:}

Lachezar Marinov MD,PhD

Department of Pediatrics

Medical University "Prof. Dr Paraskev Stoyanov"

55 Marin Drinov Str., 9000 Varna, Bulgaria

Tel.: +359/899 670 525;

E-mail:marrinovs@abv.bg 